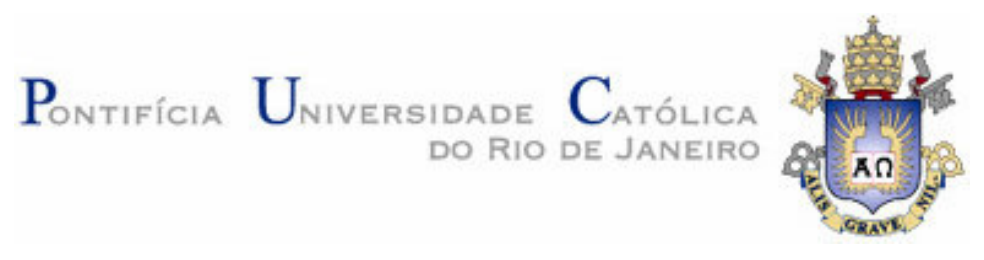

Gian Paulo Ramalho de Deus

\title{
Análise Comparativa entre a Maximização da Margem de Potência e a Minimização das Perdas Técnicas em Sistemas de
Distribuição de Energia Elétrica Potência e a Minimização das Perdas Técnicas em Sistemas de
Distribuição de Energia Elétrica
}

Dissertação de Mestrado

Dissertação apresentada como requisito parcial para obtenção do título de Mestre pelo Programa de Pós-Graduação em Engenharia Elétrica da PUC-Rio.

Orientador: Ricardo Bernardo Prada

Rio de Janeiro

junho de 2007 


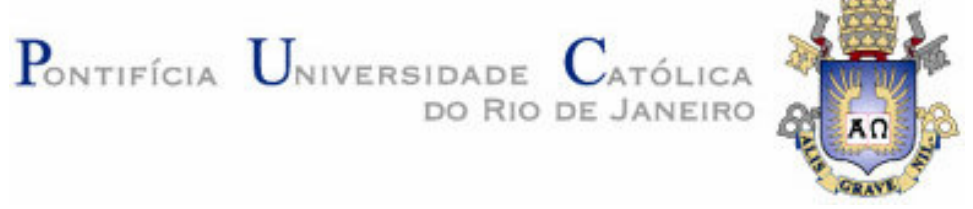

Gian Paulo Ramalho de Deus

\title{
Análise Comparativa entre a Maximização da Margem de Potência e Minimização das Perdas Técnicas em Sistemas de Distribuição de Energia Elétrica
}

Dissertação apresentada como requisito parcial para obtenção do título de Mestre pelo Programa de Pós-Graduação em Engenharia Elétrica da PUC-Rio. Aprovada pela Comissão Examinadora abaixo assinada.

\author{
Dr. Ricardo Bernardo Prada \\ Orientador \\ Departamento de Engenharia Elétrica - PUC-Rio
}

Dr. Antonio Padilha Feltrin

UNESP

Dr. Antonio Luiz Bergamo do Bomfim ELETROBRÁS

Prof. José Eugenio Leal Coordenador(a) Setorial do Centro

Técnico Científico - PUC-Rio 
Todos os direitos reservados. É proibida a reprodução total ou parcial do trabalho sem autorização da universidade, do autor e do orientador.

\section{Gian Paulo Ramalho de Deus}

Graduou-se em Engenharia Elétrica na Universidade Federal do Pará em 2004.

Ficha Catalográfica

Deus, Gian Paulo Ramalho de

Análise comparativa entre a maximização da margem de potência e a minimização das perdas técnicas em sistemas de distribuição de energia elétrica / Gian Paulo Ramalho de Deus ; orientador: Ricardo Bernardo Prada. - 2007.

91 f. : il. ; $30 \mathrm{~cm}$

Dissertação (Mestrado em Engenharia Elétrica)-Pontifícia Universidade Católica do Rio de Janeiro, Rio de Janeiro, 2007.

Inclui bibliografia

1. Engenharia elétrica - Teses. 2. Estabilidade de tensão. 3. Sistemas de distribuição. 4. Otimização. 5. Busca tabu. I. Prada, Ricardo Bernardo. II. Pontifícia Universidade Católica do Rio de Janeiro. Departamento de Engenharia Elétrica. III. Título.

CDD: 621.3 


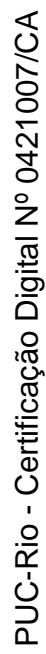

Para meus pais, Paulo Medeiros de Deus e Gertrudes Ramalho de Deus, pelo apoio e confiança. 


\section{Agradecimentos}

\section{A Deus.}

Ao meu orientador Professor Ricardo Bernardo Prada pelo estímulo e parceria para a realização deste trabalho.

À CAPES e à PUC-Rio, pelos auxílios concedidos, sem os quais este trabalho não poderia ter sido realizado.

À minha família, especialmente Lina, Karina e minhas irmãs Giselle e Girllene.

Aos meus amigos, Amanda, Jorge, David, Felipe, Fernando Machado, Leonardo Xavier e Lindomar que de uma forma ou de outra me estimularam ou me ajudaram.

Aos membros da Comissão examinadora.

A todos os amigos do curso de Pós - Graduação em Engenharia Elétrica da PUC-Rio, pelo estímulo e pela amizade.

A todos os professores e funcionários do Departamento de Engenharia Elétrica pelos ensinamentos e pela ajuda. 


\section{Resumo}

Deus, Gian Paulo Ramalho; Prada, Ricardo Bernardo. Análise Comparativa entre a Maximização da Margem de Potência e a Minimização das Perdas Técnicas em Sistemas de Distribuição de Energia Elétrica. Rio de Janeiro, 2007. 91p. Dissertação de Mestrado - Departamento de Engenharia Elétrica, Pontifícia Universidade Católica do Rio de Janeiro.

O aumento do consumo de energia elétrica leva os sistemas de distribuição a operar próximo do seu limite, podendo ocorrer situações de máximo fluxo de potência ativa e reativa nos ramos, ou seja, problemas de estabilidade de tensão. A mudança da topologia da rede permite encontrar uma configuração em que os índices de estabilidade de tensão estão distantes do ponto de máximo carregamento do sistema, reduzir as perdas técnicas e tornar a distribuição de carga nos alimentadores mais uniforme. Contudo, o número de possibilidades de chaveamento cresce com o aumento da dimensão da rede elétrica e a busca pela solução ótima requer esforço computacional elevado. O uso do algoritmo heurístico de Busca Tabu permite direcionar a busca por novas configurações de qualidade, armazenando suas características e proibindo a adição e/ou remoção de atributos por um período, evitando que a busca termine em um valor mínimo (máximo) local. Os resultados das análises em diferentes níveis de carregamento, que representam a operação do sistema radial de distribuição com carga leve, pesada e crítica, mostram que o algoritmo de Busca Tabu adotado neste trabalho consegue maximizar a margem de potência da barra crítica do sistema, levando o ponto de operação original à uma distância maior do ponto de máximo carregamento do sistema, na região normal de operação e minimizando as perdas técnicas. Com isso, evita-se que o sistema opere no ponto de máximo carregamento ou, na pior das hipóteses, na região anormal de operação, onde as ações de controle têm efeito oposto ao esperado.

\section{Palavras - chave}

Estabilidade de tensão, sistemas de distribuição, otimização, busca tabu. 


\section{Abstract}

Deus, Gian Paulo Ramalho; Prada, Ricardo Bernardo (Advisor). Comparative Analysis between the Maximization of Voltage Stability Margin and Loss Reduction in Distribution Systems. Rio de Janeiro, 2007. 91p. MSc. Dissertation - Departamento de Engenharia Elétrica, Pontifícia Universidade Católica do Rio de Janeiro.

The increase of the consumption of electric energy takes the distribution systems to operate next to its limits, which might cause situation of maximum active and reactive power flow in the branches, that is, problems of voltage stability. The change of the topology of the network allows to find a configuration where de voltage stability indexes are far from de maximum loading point, improve voltage profile, reduce power losses and load balancing. However, the number of possible switching grows with the dimension of network and searching for optimal solution requires high computational effort. Using Tabu Search heuristics allows guiding the search for new configuration of high quality, being storing its characteristics and forbidding the addition and/or removal of attributes for a period, avoiding that the search finishes in a local minimum (maximum) value. The results in different loading levels, that represent the operation of radial distribution system with normal, weighed and critical load, show that the Tabu Search algorithm maximizes the voltage stability margin, leading the original operation point to a new one that is far from the maximum loading point, in the normal region of operation. With this, it is prevented that the system operates next to the maximum loading point or, in the worse case, in the abnormal region of operation, where the actions of control have opposing effect to the waited one.

\section{Keywords}

Voltage stability, distribution systems, optimization, tabu search. 


\section{Sumário}

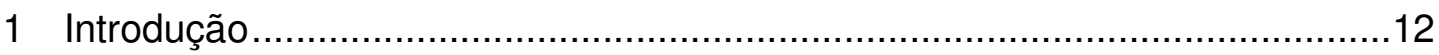

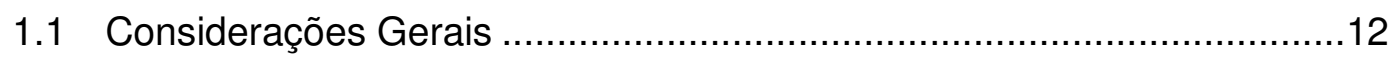

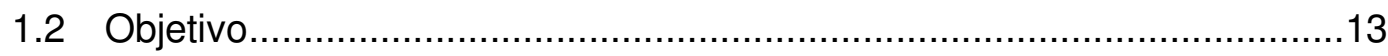

1.3 Estrutura da Dissertação ...............................................................13

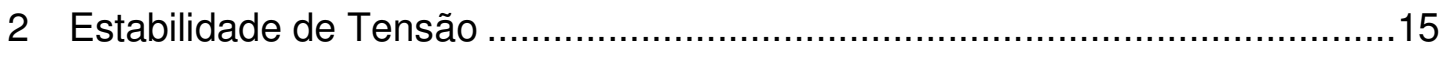

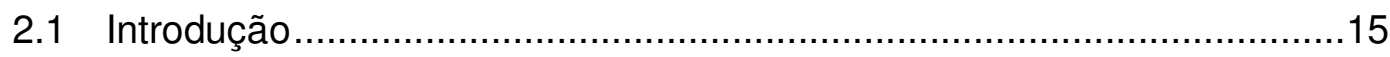

2.2 O Limite de Estabilidade de Tensão (LET) ………..............................15

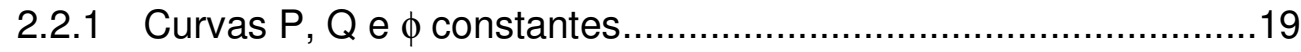

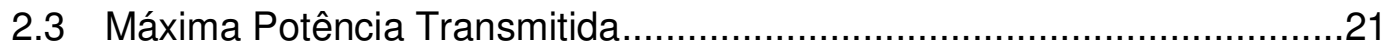

2.4 Avaliação das Condições de Estabilidade de Tensão ..............................24

2.4.1 Magnitude do determinante da matriz [D']..............................26

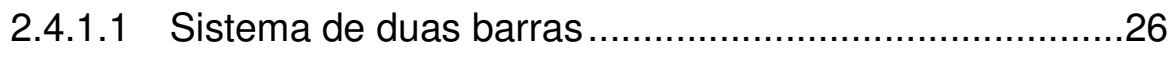

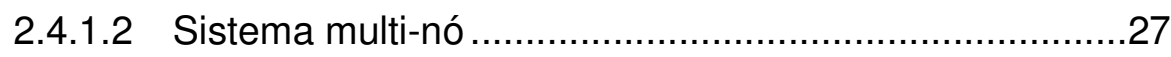

2.4.2 Sinal do determinante da matriz [D'] …….................................

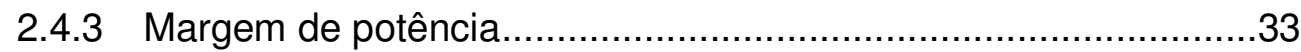

2.5 Influência do Carregamento na Margem de Potência...............................34

2.6 Índices de Estabilidade de Tensão em Redes Radiais de Distribuição ...35

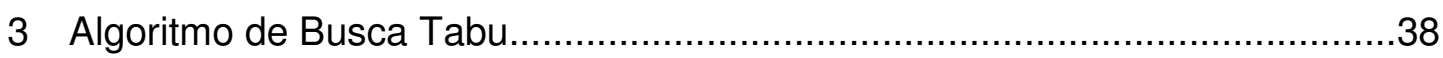

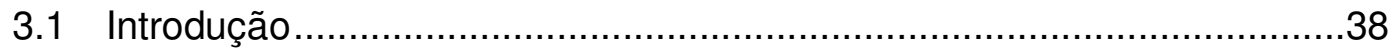

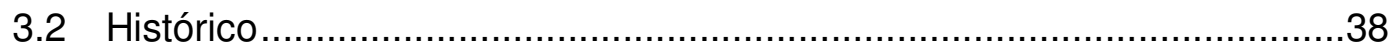

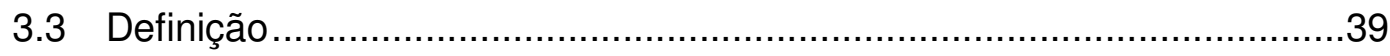

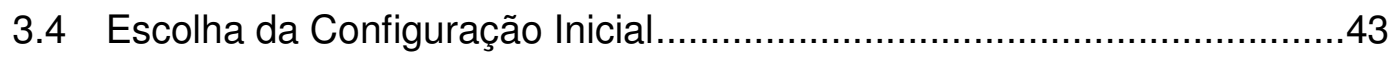

3.5 Representação e Codificação do Problema..............................................

3.5.1 Fluxo de carga em sistemas radiais de distribuição ......................43

3.5.1.1 Método de soma de correntes ......................................44

3.5.1.1.1 Cálculo das injeções de corrente nodais ................................44

3.5.1.1.2 Atualização para trás (backward sweep) ..............................45

3.5.1.1.3 Atualização para frente (forward sweep) .............................46 
3.5.1.1.4 Critério de convergência ...............................................46

3.5.1.2 Método de soma de potências..................................47

3.5.1.2.1 Atualização para frente (forward update) .............................47

3.5.1.2.2 Atualização para trás (backward update) ….........................48

3.5.1.2.3 Critério de convergência ................................................49

3.5.2 Geração das configurações vizinhas.....................................49

3.5.3 Estruturas de memória utilizadas em Busca Tabu .....................50

3.5.3.1 Memória de curto prazo .........................................51

3.5.3.2 Lista Tabu com memória de curto prazo .....................51

3.5.3.3 Memória de longo prazo .......................................52

3.5.4 Estratégias adicionais utilizadas em Busca Tabu .......................54

3.5.4.1 Configurações de elite .........................................54

3.5.4.2 Path relinking ....................................................54

3.5.4.3 Oscilação estratégica ..........................................55

3.5.5 Busca Tabu na maximização da margem de potência..................56

4 Otimização de Sistemas de Distribuição de Energia Elétrica por Busca Tabu 58 4.1 Sistema de Distribuição com Carregamento Leve ...............................58

4.2 Sistema de Distribuição com Carregamento Pesado ...........................68

4.3 Sistema de Distribuição com Carregamento Crítico ............................77

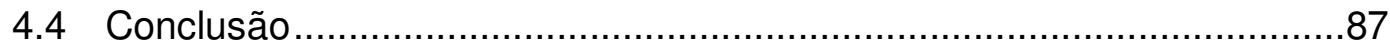

5 Conclusões Finais e Propostas para Trabalhos Futuros …..........................88

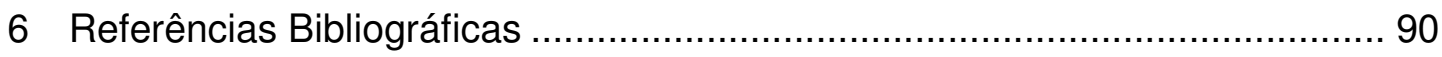




\section{Lista de Tabelas}

Tabela 2.1 - Margem de potência em função do aumento do carregamento ........34

Tabela 4.1 - Índices de estabilidade de tensão no carregamento leve..................59

Tabela 4.2 - Carregamento Leve............................................................60

Tabela 4.3 - Aumento da margem de potência no carregamento leve ..................62

Tabela 4.4 - Índices de estabilidade de tensão carregamento leve.......................64

Tabela 4.5 - Redução das perdas técnicas no carregamento leve.......................66

Tabela 4.6 - Distância ao máximo carregamento das barras crítica e adjacentes68

Tabela 4.7 - Índices de estabilidade de tensão no carregamento pesado.............69

Tabela 4.8 - Carregamento Pesado ....................................................... 70

Tabela 4.9 - Aumento da margem de potência no carregamento pesado.............71

Tabela 4.10 - Índices de estabilidade de tensão no carregamento pesado ..........73

Tabela 4.11 - Redução das perdas técnicas no carregamento pesado.................75

Tabela 4.12 - Índices de estabilidade de tensão no carregamento crítico ............78

Tabela 4.13 - Carregamento Crítico …....................................................... 79

Tabela 4.14 - Aumento da margem de potência no carregamento crítico .............81

Tabela 4.15 - Ponto de operação após maximização da margem de potência ....84

Tabela 4.16 - Redução das perdas técnicas no carregamento crítico.................. 85 


\section{Lista de Figuras}

Figura 2.1 - Circuito de duas barras do limite de estabilidade de tensão ..............16

Figura 2.2 - Circuito de duas barras da potência injetada na barra terminal .........19

Figura 2.3 - Curvas $P$ e $Q$ constantes no plano $\theta_{1}$ versus $V_{1}$ na barra terminal ....22

Figura 2.4 - Curva PV referente ao sistema de duas barras ............................23

Figura 2.5 - Localização do Vetor Gradiente de $P_{i}$ e $Q_{i}$ no plano $V \theta$...................31

Figura 2.6 - Magnitude do $\operatorname{det}[\mathrm{A}]$ do sistema e do $\operatorname{det}[\mathrm{D}$ '] de uma barra remota. ..32

Figura 2.7 - Sinal da margem de potência na curva PV .....................................34

Figura 3.1 - Pseudo-código genérico do método de descida simples .................40

Figura 3.2 - Transição de configurações no algoritmo de Busca Tabu .................42

Figura 3.3 - Espaço de busca entre busca local e Busca Tabu..........................42

Figura 3.4 - Sistema radial de distribuição de cinco barras ...............................44

Figura 3.5 - Laço formado ao se fechar a chave i-j .....................................50

Figura 3.6 - Exemplo de armazenamento da Lista Tabu em vetor........................52

Figura 3.7 - Intensificação e diversificação em Busca Tabu ..............................53

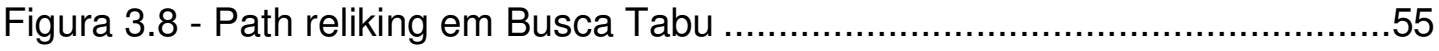

Figura 3.9 - Pseudo-código Busca Tabu aumento da margem de potência .........57

Figura 4.1 - Diagrama unifilar do sistema de distribuição ..................................61

Figura 4.2 - Maximização da margem de potência no carregamento leve ...........62

Figura 4.3 - Diagrama unifilar após o aumento da margem (Leve) ......................63

Figura 4.4 - Perfil de tensão antes e depois da reconfiguração (Leve) .................65

Figura 4.5 - Minimização das perdas técnicas com carregamento leve ...............66

Figura 4.6 - Diagrama unifilar após a redução das perdas técnicas (Leve)..........67

Figura 4.7 - Maximização da margem de potência com carregamento pesado ...71

Figura 4.8 - Perfil de tensão antes e depois da reconfiguração (Pesada) .............72

Figura 4.9 - Diagrama unifilar após o aumento da margem (Pesado) ...................74

Figura 4.10 - Minimização das perdas técnicas com carregamento pesado ..........76

Figura 4.11 - Maximização da margem de potência com carregamento crítico....80

Figura 4.12 - Diagrama unifilar após o aumento da margem (Crítico) ..................82

Figura 4.13 - Perfil de tensão antes e depois da reconfiguração (Crítico).............83

Figura 4.14 - Diagrama unifilar após a redução das perdas técnicas (Crítico) .... 86 